at once the very marked similarity between tho excretory vesicle of young adults of this species of Renicola and that of the Rhodometopa group of cercariæ, particularly $C$. pythionike Rothschild ${ }^{2}$. In both cases the vesicle is $\boldsymbol{Y}$-shaped with numerous lateral branches and with a connecting channel joining the two arms of the $Y$ immediately behind the ventral sucker.

Evidence of this kind seems fairly strong, and furth $\in \mathbf{r}$ work is being carried out in the hope that a knowledge of the larval stages may help to establish more definitely the true systematic position of this somewhat disputed genus.

Department of Zoology,

$$
\text { C. A. Wright }
$$

Imperial College of Science and Technology, London, S.W.7. Nov. 18.

${ }^{1}$ Campbell and Sloane, Vet. J., 99, 291 (1943).

${ }^{2}$ Rothschild, M., Parasitology, 27, 152 (1935).

\section{Relationship between the Oxidative and Fermentative Phases during Adaptation to Galactose in Saccharomyces cerevisiz}

Afrobic adaptation to the utilization of galactose in Saccharomyces cerevisioe occurs in two phases : an oxidative phase in which the respiratory quotient is 1 , and a fermentative phase which follows it ${ }^{1}$. Further experiments have now demonstrated that during the oxidative phase of adaptation to galactose, the yeast cells are capable of fermenting galactose if anaerobic conditions are introduced. The degree of fermentation which resulted when anaerobic conditions were instituted was found to be correlated with the degree of adaptation during the oxidative phase as measured by the $Q_{\mathrm{CO}_{2}}$.

These results confirmed previous ones which indicated that adaptation to the utilization of galactose is not concerned with oxidation or fermentation as such, but rather with an enzyme or series of enzymes which make galactose available to the cell for subsequent oxidation or fermentation ${ }^{1}$. Since the oxidative phase of aerobic adaptation was always followed by the onset of fermentation, provided the concentration of galactose was sufficiently high, it was of extreme interest to determine the factors involved in this shift from oxidation to fermentation under aerobic conditions.

It was found that, during the early stages of adaptation long before fermentation started, carbohydrate was being stored, some as glycogen and some as glycolytic intermediates, and pyruvate did not accumulate appreciably until storage of glycolytic intermediates was close to maximum. Therefore, it appears that storage occurs as soon as adaptation starts, and that only a relatively small amount of galactose is initially metabolized to pyruvic acid and oxidized through the tricarboxylic acid cycle. However, when storage is at a maximum and adaptation has proceeded sufficiently, galactose is metabolized at a greater rate to pyruvate until the oxidative mechanisms are apparently saturated, and pyruvate and acetaldehyde accumulate. It is at this point that fermentation starts. These conclusions are in agroement with previous results which demonstrated that, at low concentrations of galactose, adaptation occurred readily (as indicated by increase in $Q_{0}$, disappearance of galactose, and accumulation of glycogen) but that fermentation did not occur ${ }^{1}$.
An important implication of these results is that fermentation is not a valid criterion for measuring the synthesis of the galactose-adaptive enzyme. The onset of galactose fermentation in yeast appears to be dependent upon the preliminary accumulation of reserves and intermediates, and only in a superficial way related to the kinetics of adaptive enzyme formation. Therefore, experiments using this method which purport to show that the synthesis of galactokinase is autocatalytic in nature are invalid ${ }^{2}$, and theories which postulate the existence of extrachromosomal hereditary factors in order to explain the kinetics of galactokinase formation in yeast ${ }^{2}$ are premature.

I wish to thank Dr. Carl C. Lindegren for encouragement during the progress of the work. This work has been supported by grants from the American Cancer Society, administered by the Committee of Growth, National Research Council, National Cancer Institute of the National Institutes of Health, Public Health Service and Anheuser-Busch, Inc.

A. LeONARD SHEFFNER

Southern Illinois University, Carbondale, Illinois.

$$
\text { Nov. } 11 .
$$

${ }^{1}$ Sheffner, A. Jeonard, and Lindegren, Carl C., J. Bact., 64, 423 (1952). 2 Spiegelman, S., Symp. Soc. Exp. Biol., No. 2, Growth, 286 (1948).

\section{Purification and Cellulolytic Activity of Cellvibrio}

IN recent years, several workers have paid a great deal of attention to the purification and the cellulolytic activity of aerobic cellulose-decomposing bacteria, especially Cytophaga and Cellvibrio. Isolations have generally been carried out by direct plating of soil suspensions on precipitated cellulose agar, and by enrichment cultures on silica-gel filter paper or on filter paper partly immersed in a liquid mineral medium. With the former method, most of the dishes are invaded by moulds and generally the formation of lytic zones is not very obvious.

In order to isolate Cellvibrio, a first enrichment was effected on silica-gel filter paper. After tenfold subculturing on the same medium, impurities in the form of other bacteria were still present. The impure strains were plated out on precipitated cellulose agar containing the common minerals, $0 \cdot 1$ per cent farmyard manure extract and 0.2 per cent cellulose. After seven days incubation at $26^{\circ} \mathrm{C}$, different types of colonies were obtained. Some were surrounded by clear lytic zones while other colonies were not. All the Cellvibrio colonies that produced lytic zones on this medium showed a luxuriant growth when subcultured on filter paper agar. The strains obtained in this way were again plated on cellulose agar until only one type of colony with clear lytic zones was present. These strains were considered to be absolutely pure.

Not all strains of Cellvibrio, after enrichment on filter paper, produced lytic zones on precipitated collulose agar to which farmyard manure extract was added. Some strains need other growth factors in addition to those present in the farmyard manure extract. More work will be done on the role of these factors in the formation of lytic zones by Cellvibrio on cellulose agar.

'The pure cultures already have been maintained during four months by alternate subculturing on agar filter paper and precipitated cellulose agar provided with farmyard manure extract. 